\title{
THE INVESTIGATION OF IONOSPHERIC CHANNEL INFLUANCES FOR PHASED SHIFT KEYING DIGITAL SIGNALS
}

\section{Lev E. Nazarov, Vitaly V. Batanov}

Kotelnikov Institute of Radioengineering and Electronics of RAS, Fryazino Branch, http:// fire.relarn.ru Fryazino 141190, Moscow Region, Russian Federation

nazarov@ire.rssi.ru,batanov@mail.ru.

Abstract. The results of analysis for signals propagating through the ionospheric satellite communication channels are presented in the article. As a measure of distortion estimates concerning to the free space propagating the signal/noise degradation and the detection errorperformances are used. The descriptions of signal distortions due to ionospheric influence are based on the time-domain solution for ionospheric channel as linear stationary filter. The signal/ noise and error-performance degradations for two-phased shift keying signals are evaluated. The computer simulations for evaluation of these error-performance degradations concerning signal/ noise decreasing are performed - the degradation of signal/noise is about $2.5 \mathrm{~dB}$ for bit-error 0.00001 .

Keywords: ionosphere, satellite ionospheric channel, error-performances, signals, phase shift keying signals

UDC 621.391.01

Bibliography - 15 references

Received 26.03.2019, accepted 31.03.2019

RENSIT, 2019, 11(1):65-72

DOI: 10.17725/rensit.2019.11.065

\section{CONTENTS}

1. Introduction (65)

2. Problem statement (66)

3. IONOSPHERE MODELS (67)

4. Description of digital DISTORTION (67)

5. Calculation Results (69)

6. Conclusion (70)

REFERENCES (71)

\section{INTRODUCTION}

The ionosphere is a heterogeneous dispersive propagation medium, which causes phasefrequency and amplitude-frequency distortions of digital signals during their propagation [1-4]. These distortions lead to intersymbol interference (ISI) in addition to additive channel thermal noise (AWGN) and energy losses during correlation signal processing, which is the basis of signal detection procedures during synchronization and demodulation of signals in digital communication systems with respect to propagation in free space [5-7].

The problem of studying and quantifying the distortion of digital signals with phase shift keying with the spread of the spectrum during their propagation through ionospheric radio links for satellite communication systems and satellite navigation systems is a pressing problem [5-9]. Quantitative estimates of the distortion data are made on the basis of a comparative analysis of the probability characteristics when receiving distorted signals and undistorted signals and depend on the working frequency ranges $[1,4]$. In this article, these estimates are given for the P-band radio links, which are extensively used in the development and creation of a number of satellite information systems with the expansion of the spectrum of signals for various purposes, for example, in developing the second-generation 
Cospas-Sarsat international satellite search and rescue system (center frequency 406.1 $\mathrm{MHz}$ ) [10].

\section{PROBLEM STATEMENT}

The basis of methods for analyzing the influence of the ionosphere on digital signal distortions is the solution of the wave equation during propagation along the $\mathrm{z}$ axis of a plane wave $\mathrm{E}(\mathrm{z}, \mathrm{f})$ with a frequency $f$ normally incident on a layer of inhomogeneous medium with a dielectric constant $\varepsilon(\mathrm{z}, \mathrm{f})[2,6]$

$$
\frac{d^{2} E(z, f)}{d z^{2}}+\frac{(2 \pi)^{2} f^{2}}{c^{2}} \varepsilon(z, f) E(z, f)=0 \text {. }
$$

Here $\mathrm{c}$ is the speed of light in a vacuum.

Wave equation (1) determines the particular model of radio wave propagation in the direction of the Earth's magnetic field considered below. In this case, only the polarization plane of the radio waves rotates during propagation [3, 4]. An alternative model is the propagation of radio waves in a direction perpendicular to the direction of the Earth's magnetic field, which takes into account two-beam propagation (the occurrence of ordinary and extraordinary waves) and, in addition to the distortions under consideration, also fading signals (frequency-selective and nonselective frequency) [3]. This model assumes independent research.

A number of analytical solutions of equation (1) were obtained using simplifications with respect to $\varepsilon(\mathrm{z}, \mathrm{f})$, assuming, in particular, the propagation medium is isotropic (the model of "cold" plasma), as well as flat-layered or spherically layered [2]. An important direction is the determination of approximate solutions of the wave equation (1) using these simplifications, in particular, the approximation of geometric optics. A sufficient condition for the applicability of this approximation is the slowness of changes in the dielectric properties of the medium at wavelength $\lambda$, i.e. $[2,4]$. In this case, the approximation of geometric optics to the solution of the wave equation without taking into account the reflection for the inhomogeneous ionosphere has the form [2] $E(z, t)=E(0, t) \exp \left(\frac{j 2 \pi f}{c} \int_{0}^{z} n(z, f) d z\right)$.

Here is the refractive index of the medium.

Relation (2) is valid for monochromatic waves. For digital signals with center frequency $\mathrm{f} 0$, specified as [5]

$$
E(0, t)=\dot{A}(t) \exp \left(j 2 f_{0} t\right)
$$

and representing a spectrum of monochromatic waves, the task becomes more complicated. In this case, each spectral component during propagation acquires a partial phase shift and a partial change in amplitude, which causes the resulting distortion of signals at the input of the receiving device. In (3), the designation corresponds to the complex envelope of the signals defined by the signal "constellation" with the duration $\mathrm{T}$ of the constituent elements with phase or quadrature amplitude manipulation [5].

The essence of the task is to describe the distortions of signals during their propagation through satellite ionospheric radio links and to make an assessment of the influence of these distortions on the probabilistic characteristics when receiving signals.

\section{MODELS OF THE IONOSPHERE}

For frequencies $f$ used in satellite communication systems ( $\mathrm{f}>30 \mathrm{MHz}$ [1]), the dielectric constant of the ionosphere $\varepsilon(z, f)$ in the form of a heterogeneous spherically 
symmetric medium is given by the relation [ 1 , 3, 4]

$$
\varepsilon(z, f)=1-f_{p}^{2}(z) / f^{2} \text {. }
$$

Here is the natural frequency of the ionosphere $(\mathrm{kHz}) ; \mathrm{Ne}(\mathrm{z})$ is the electron density of the ionosphere $(\mathrm{el} / \mathrm{cm} 3)$ at height $z$.

This model of the dielectric constant $\varepsilon(z$, f) (4) is real, the phenomena of reflection, scattering and absorption of radio waves are assumed to be small. This causes only phase-frequency distortion during signal propagation [10].

The electron density of the ionosphere $\mathrm{Ne}$ (z) depends on the altitude, latitude and longitude, on the time of day and solar activity. A number of papers $[1-4,11]$ are devoted to the development and development of its models. These models are used to solve particular problems of navigation, communication, radio wave propagation, etc. Below, the calculations use the well-known and simplest model (one-layer Chapman model) for $\mathrm{Ne}(\mathrm{z})$ [3]

$$
N_{e}(z)=\sqrt{\gamma \exp \left[\left(\exp \left(b\left(z-z_{\text {max }, e}\right)\right)+b z\right)\right]} .
$$

Here $\gamma, b$ are the model parameters; zmax, $\mathrm{e}$ is the height above the earth's surface, for which the maximum value of the electron density is reached. For daytime, the maximum value of $\mathrm{Ne}(\mathrm{z})=106 \mathrm{el} / \mathrm{cm} 3$ [3] (model parameters $\mathrm{b}=0.01, \gamma=5 \cdot 1013$, zmax, e $=300 \mathrm{~km}$ ), the corresponding value of the natural frequency of the ionosphere for zmax, e is equal to $\mathrm{fp}=9 \mathrm{MHz}$.

The use of model (5) makes it possible to determine approximate distortions of signals when variations of its parameters (in particular, when quantifying distortions for the most pronounced effect of the ionosphere in the daytime). To further refine the quantitative estimates of the distortions, it is necessary to use more complex particular models of the ionosphere, for example, the IRI statistical models and its modifications [11].

\section{DESCRIPTIONS OF DISTORTIONS OF DIGITAL SIGNALS}

Methods for analyzing the distortions considered during the propagation of digital signals belong to two general classes $[2,6$, 9]. The first class includes the methods of analyzing the distortions in the time domain using the impulse response $h(z, \tau)$ of the ionospheric line $[12,13,14]$. The second class includes methods for analyzing distortions in the frequency domain using the Fourier spectral transform apparatus for $h(z, \tau)[2$, $6,15]$.

The representation of the signal $E(z, t)$ using the impulse response of the ionospheric line is given by the convolution ratio [13] $E(z, t)=\int_{0}^{\infty} E(0, \tau) h(z, t-\tau) d \tau$.

Distortion analysis methods from the second class are based on the calculation of the inverse Fourier transform $[2,6]$

$$
E(z, t)=\int_{-\infty}^{\infty} g(f) \exp (j(\varphi(f, z)+2 \pi f t)) d f .
$$

Here $g(f)$ is the signal spectrum; - phase of the spectral component for frequency $f$; wave number.

The impulse response $h(z, \tau)$ in (6) for a stationary inhomogeneous ionosphere can be represented as an approximate analytical expression. One of such expressions is given in [13]

$$
\begin{aligned}
& h\left(z, \tau^{\prime}\right)=\delta\left(\tau^{\prime}\right)- \\
& -u\left(\tau^{\prime}\right) \frac{2 \pi f_{\mathrm{p}, \text { eff }}(z, f) \sqrt{z}}{\sqrt{2 c \tau^{\prime}}} J_{1}\left(4 \pi f_{\mathrm{p}, \mathrm{eff}}(z, f) \sqrt{\frac{z \tau^{\prime}}{2 c}}\right) .
\end{aligned}
$$


Here $\delta(\tau)$ is the delta function; $\tau{ }^{\prime}=\tau-\mathrm{z}$ / c; $\mathrm{u}(\tau)$ is the unit jump function; - Bessel function

nth order. For a heterogeneous ionosphere, its effective value of the natural frequency $\mathrm{fp}$, eff for $\mathrm{fO}$ is defined as a solution to the equation

$$
\int_{0}^{z} \sqrt{f_{0}^{2}-f_{p}^{2}(x, f)} d x=z \sqrt{f_{0}^{2}-f_{p, e f f}^{2}} .
$$

When calculating (9) and the $\varphi(f, z)$ phase in (7), the Ne ( $\mathrm{z}$ ) profile model (5) is used.

For the frequency range of satellite radio transmission lines, the condition $\mathrm{fO}>>\mathrm{fp}(\mathrm{z}$, $\mathrm{f}$ ) is fulfilled and the calculation of $\mathrm{fp}$, eff can be performed using the relation. The effective natural frequency fp, eff is practically independent of the center frequency $\mathrm{fO}$ and is functionally related to the total electronic content This parameter is one of the main characteristics of the Earth's ionosphere [3, 4].

In [14] the second analytical expression is given relative to

$$
\begin{aligned}
& h(z, \tau)=\delta(\tau-z / c)- \\
& -u(\tau-z / c) \frac{2 \pi f_{p, e f f} z J_{1}\left(2 \pi f_{p, e f f} \sqrt{\tau^{2}-(z / c)^{2}}\right)}{c \sqrt{\tau^{2}-(z / c)^{2}}} .
\end{aligned}
$$

For radio lines of satellite digital communication systems, the condition $\mathrm{T}<<$ $\mathrm{z} / \mathrm{c}$ is satisfied; in this case, the identity of expressions (8) and (10) was proved in [14].

Relations (8) and (10) correspond to the elevation angle $\theta=90^{\circ}$ of the receiving device on board the spacecraft with respect to the transmitting device on the earth's surface. For an arbitrary elevation angle in (10), the zincl slant range is used, calculated using the ratio

In fig. 1 shows the form of the second term of the characteristic $h(z, \tau)(10)$, excluding the delta function and the sign.

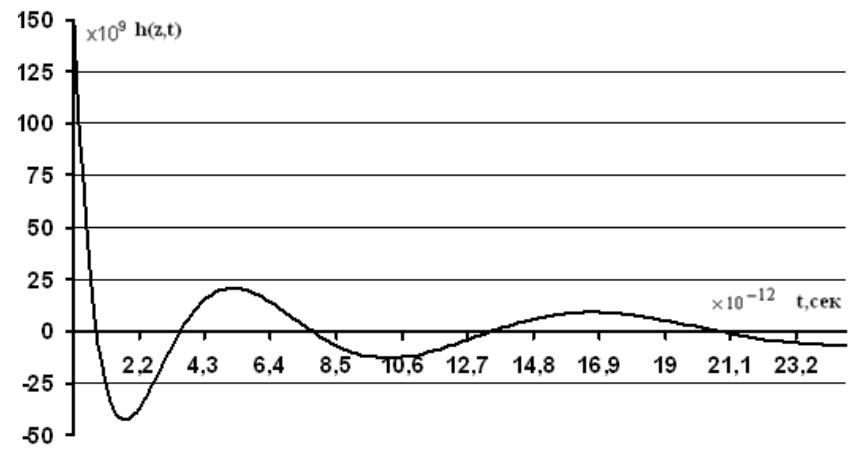

Рис. 1. Вид слагаемого импульсной характеристики $b(2, \tau)$ (высота $z=400 \mathrm{kM}, f_{p, e f f}=3.5 \mathrm{M \Gamma u}$ ).

The curve is calculated for a height of $\mathrm{z}=$ $400 \mathrm{~km}$, fp, eff $=3.5 \mathrm{MHz}, \theta=90^{\circ}$. It can be seen that the characteristic $h(z, \tau)$ has a sufficiently large length, which determines the aftereffect of the distorted constituent elements and, as a consequence, the presence of ISI in the transmission of digital signals.

In fig. 2 shows the form of the initial element of a digital signal with two-phase (FM2) manipulation with the envelope in the form of a meander (curve 1), the center frequency of the radio pulse is $\mathrm{fO}=400$ $\mathrm{MHz}$, the duration is $\mathrm{T}=50$ ns. Curve 2 is calculated using relation (10) and corresponds to this element when propagating in the ionosphere, characterized by the effective value of the natural frequency $\mathrm{fp}$, eff $=3.5$ $\mathrm{MHz}$, the height of the ionosphere above the earth's surface is $z=400 \mathrm{~km}$. It can be seen that the distorted element has the radio pulse repetition noted above. The difference in the time of appearance of the initial pulse $\mathrm{E}(0$,

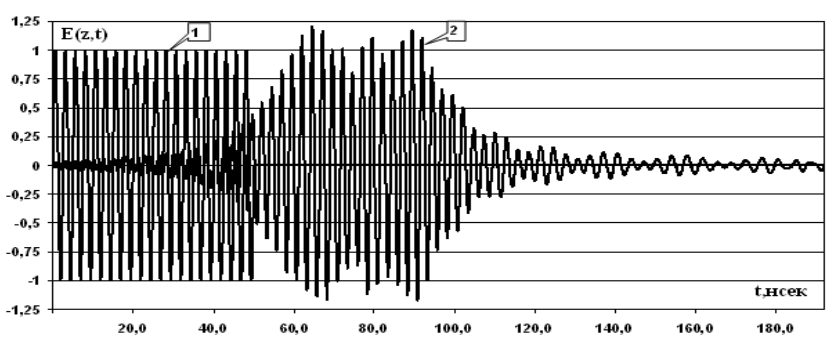

Рис. 2. Вид элемента чифрового сигнала ФМ2: кривая 1 исходньии элемент $\left(f_{0}=400 \mathrm{MГ \nu ,} \mathrm{T}=50\right.$ нс); кривая 2 - вид элемента при распространении в ионосфере $\left(f_{p, e f f}=3.5 \mathrm{MГ}\right.$, $z=400 \mathrm{\kappa m})$. 
t) for the model of propagation in free space and the pulse $E(z, t)$ at the output of the ionospheric radio link is due to the difference between the group velocity and the speed of light $\mathrm{c}>$ vgr.

The distortions of the constituent elements $E(z, t)$ cause the presence of energy losses $\Delta \mathrm{E}$ with respect to propagation in free space. These losses are due to a change in the envelope of the distorted signals with a variation in their power and the occurrence of interference by the ISI $[12,14]$.

The energy loss $\Delta \mathrm{E}$ is estimated on the basis of a comparative analysis of the probabilities of erroneous reception of the $\mathrm{Pb}$ information bits for signals without distortion and with distortions.

For the basic model of channel noise AMGSH, the optimal reception rule is based on the calculation of the cross-correlation of the input realization with the original signals, the probability of an incorrect $\mathrm{Pb}$ reception for the FM2 signals is determined by the relation [5]

$$
P_{b}=1-F \sqrt{2 E_{b} / N_{0}} \text {. }
$$

Here, $\mathrm{Eb}$ is the energy of signals per information bit, $\mathrm{N} 0$ is the ABGSh spectral density (one-sided),

$$
F(x)=\frac{1}{\sqrt{2 \pi}} \int_{-\infty}^{x} \exp \left(-t^{2} / 2\right) d t
$$

\section{RESULTS OF CALCULATIONS}

Below are the results of estimating the energy loss $\Delta \mathrm{E}$ when digital signals propagate with two-phase ("constellation" FM2) and fourphase ("constellation" FM4) manipulation on the ionospheric radio link with parameters of the daytime ionosphere model (5) - height $\mathrm{z}=400 \mathrm{~km}$, central frequency $\mathrm{fO}=400 \mathrm{MHz}$. These signals are basic for the development of satellite information systems for various purposes $[5,8,10]$. Variable parameters - the

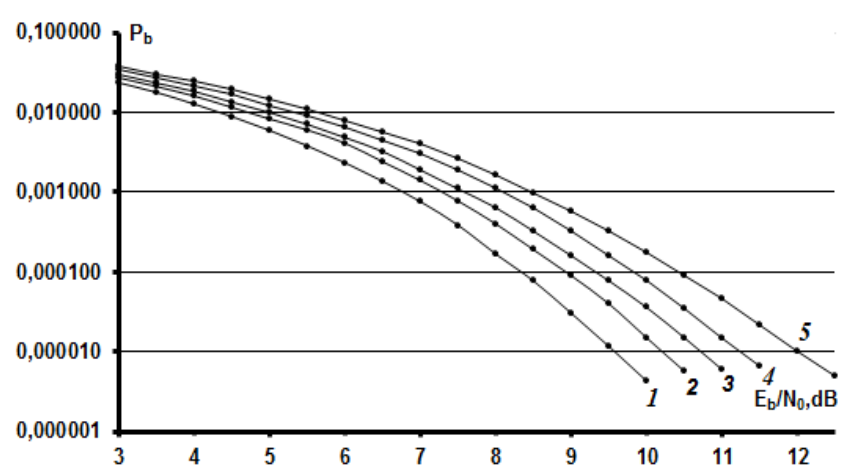

Рис. 3. Вероятности ошибочного приема сигналов ФМ2 ири распространении по ионосферной минии, $f_{0}=400 \mathrm{MГ}$, z = 400 км: 1 - распространение в свободном пространстве; 2 - $\theta$ $=90^{\circ}, \mathrm{T}=100 \mathrm{Hc}, \Delta \mathrm{F}=20 \mathrm{M \Gamma} ; 3-\theta=10^{\circ}, \mathrm{T}=100 \mathrm{Hc}$, $\Delta F=20 M \Gamma y ; 4-\theta=90^{\circ}, T=50 \mathrm{Hc}, \Delta F=40 \mathrm{M \Gamma} ; 5-\theta$ $=10^{\circ}, \mathrm{T}=50 \mathrm{Hc}, \Delta \mathrm{F}=40 \mathrm{M \Gamma} \mathrm{y}$.

duration of the elements of the signals $\mathrm{T}$, the frequency band of the signals $\Delta \mathrm{F}=2 / \mathrm{T}$, the signal-to-noise ratio $\mathrm{Eb} / \mathrm{N} 0$; elevation angle $\theta$.

The purpose of the simulation was to calculate approximate estimates of $\Delta \mathrm{E}$ for the most pronounced effect of the ionosphere in the daytime. These estimates must be taken into account when calculating the energy budget of the created P-frequency satellite radio links, an example is the secondgeneration Cospas-Sarsat satellite system being developed [10].

In Fig. 3 and Fig. 4 shows the dependences of the probabilities of $\mathrm{Pb}$ error on $\mathrm{Eb} / \mathrm{NO}$

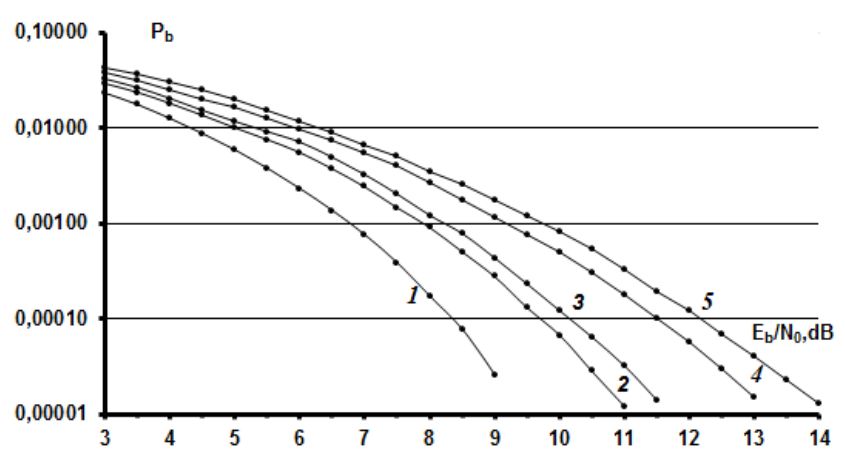

Рис. 4. Вероятности ошибочного приема сигналов ФМ4 ири распространении по ионосферной минии, $f_{0}=400 \mathrm{M \Gamma}$, z. 400 км: 1 - распространение в свободном пространстве; 2 - $\theta$ $=90^{\circ}, \mathrm{T}=100 \mathrm{Hc}, \Delta \mathrm{F}=20 \mathrm{M \Gamma} ; 3-\theta=10^{\circ}, \mathrm{T}=100 \mathrm{Hc}$, $\Delta F=20 M \Gamma y ; 4-\theta=90^{\circ}, T=50 \mathrm{Hc}, \Delta \mathrm{F}=40 \mathrm{M \Gamma}$; $5-\theta$ $=10^{\circ}, \mathrm{T}=50 \mathrm{Hc}, \Delta \mathrm{F}=40 \mathrm{M \Gamma y}$. 
for the FM2 and FM4 signals, calculated by computer simulation of the reception algorithm based on correlation ratios.

In modeling the reception of signals, an interval estimate of the probability of $\mathrm{Pb}$ was made by calculating the frequency $\mathrm{w}=\mathrm{x} / \mathrm{u}$. Here $\mathrm{x}$ is the number of erroneous decisions in a sequence of independent computational experiments of volume $\mathrm{u}$, determined by the size of the confidence interval, the probability $\mathrm{Pb}$, the confidence probability Pconf. For example, for $P_{b}=10^{-5}, \alpha=0.5 \mathrm{~Pb}$ (confidence interval $[0.5 \mathrm{~Pb}, 1.5 \mathrm{~Pb}]$ ) and Pconf $=0.95$, the required number of experiments is $\mathrm{u}>$ 1540000.

Curve 1 in fig. 3 is calculated using relation (11) and corresponds to the propagation of FM2 signals in free space — the probability $P_{b}=10^{-5}$ is ensured at the ratio $E_{b} / N_{0}=9.5$ dB. Curves 2, 3, 4, 5 are obtained by modeling and correspond to the propagation along the ionospheric radio link. Curves 2 and 3 correspond to the duration $\mathrm{T}=100 \mathrm{~ns}(\Delta \mathrm{F}$ $=20 \mathrm{MHz}$ ) and the elevation angles $\theta=90^{\circ}$ and $\theta=10^{\circ}$. Curves 4 and 5 correspond to the duration $\mathrm{T}=50 \mathrm{~ns}(\Delta \mathrm{F}=40 \mathrm{MHz})$ and elevation angles $\theta=90^{\circ}$ and $\theta=10^{\circ}$.

The summary Table shows the corresponding energy loss estimates $\Delta \mathrm{E}$ for $\mathrm{Pb}=10-5$. One can see a monotonous decrease in the probability of $\mathrm{Pb}$ to 0.000001 depending on $\mathrm{Eb} / \mathrm{N} 0$, which shows a rather small influence of AMI interference on the

Таблица

Оценки энергетических потерь $\Delta \mathrm{E}$ при распространении сигналов ФМ2 и ФМ4 по ионосферной радиолинии относительно распространения в свободном пространстве $\left(P_{h}=10^{-5}\right)$.

\begin{tabular}{|c|c|c|c|}
\hline \multicolumn{2}{|c|}{ Параметры } & $T=100 \mathrm{HC}$ & $T=50 \mathrm{HC}$ \\
\hline \multirow{2}{*}{ ФМ2 } & $\theta=90^{\circ}, \Delta E_{\triangle} \mathrm{Б}$ & 0.5 & 1.0 \\
\cline { 2 - 4 } & $\theta=10^{\circ}, \Delta E_{A} \mathrm{Б}$ & 1.5 & 2.5 \\
\hline \multirow{2}{*}{ ФM4 } & $\theta=90^{\circ}, \Delta E_{A} \mathrm{Б}$ & 1.5 & 3.5 \\
\cline { 2 - 4 } & $\theta=10^{\circ}, \Delta E_{A} \mathrm{Б}$ & 2.0 & 4.5 \\
\hline
\end{tabular}

probability characteristics in this case relative to the effect due to the energy variations of the elements of the digital signals. It is also seen that with a decrease in the duration $\mathrm{T}$ (an increase in the frequency band $\Delta \mathrm{F}$ ), an increase in $\Delta \mathrm{E}$ values is observed - at $\theta=0^{\circ}$, we have $\Delta \mathrm{E}=0.5 \mathrm{~dB}$ for $\mathrm{T}=100 \mathrm{~ns}$ and $\Delta \mathrm{E}$ $=1.0 \mathrm{~dB}$ for $\mathrm{T}=50 \mathrm{~ns}$; at $\theta=10^{\circ}$, we have $\Delta \mathrm{E}=1.5 \mathrm{~dB}$ for $\mathrm{T}=100 \mathrm{~ns}$ and $2.5 \mathrm{~dB}$ for $\mathrm{T}=50 \mathrm{~ns}$.

In fig. 4 shows the probability curves for the signals FM4. Curve 1 is also calculated using relation (11) and corresponds to propagation in free space. Curves $2,3,4,5$, 6 are obtained by modeling and correspond to the propagation along the ionospheric radio link. Curves 2 and 3 correspond to the duration $\mathrm{T}=100 \mathrm{~ns}(\Delta \mathrm{F}=20 \mathrm{MHz})$ and angles $\theta=90^{\circ}$ and $\theta=10^{\circ}$. The summary table shows the corresponding estimates of the energy loss $\Delta \mathrm{E}$ for FM4 signals at $\mathrm{Pb}=$ 10-5. It can be seen that with respect to the signals of the FM2, the energy losses increase - at $\mathrm{Pb}=10-5$, the loss of $\Delta \mathrm{E}$ with respect to curve 1 reaches 1.5 and $2.0 \mathrm{~dB}$. Curves 4 and 5 correspond to the duration $\mathrm{T}=50 \mathrm{~ns}(\Delta \mathrm{F}$ $=40 \mathrm{MHz}$ ) and angles $\theta \mathrm{A}=90^{\circ}$ and $\theta \mathrm{A}=$ $10^{\circ}$. In this case, the $\Delta \mathrm{E}$ losses also increase and reach 3.5 and $4.5 \mathrm{~dB}$, which shows an increase in the influence of AMI interference and variations in the energy of digital signal elements on the probability characteristics of reception with respect to the FM2 signals.

\section{CONCLUSION}

Methods are given for describing digital signals as they propagate through the ionospheric radiolinks of satellite information transmission systems. Description methods are based on the use of a stationary ionospheric radio link model in the form 
of a linear filter with an impulse response represented by analytical expressions.

The results of the quantitative estimation of the energy losses when receiving the distorted signals FM2 and FM4 when they propagate through radio links of the P-frequency range relative to the propagation in free space are given. By modeling, it was shown that the energy losses depend on the duration of the digital signal elements (frequency band of signals) and on the elevation angle and reach $2.5 \mathrm{~dB}$ and 4.5 $\mathrm{dB}$, respectively, for FM2 signals and FM4 signals for the most pronounced effect of the ionosphere in the daytime, which must be taken into account when calculating energy budget of satellite radio links.

Estimation of energy losses using more complex models of the height profiles of the electron density of the ionosphere, taking into account, in particular, the twobeam propagation of signals with different "constellations", represents a promising line of research.

\section{REFERENCES}

1. Kolosov MA., Armand NA, Yakovlev OI. The propagation of radio waves in space communications. M., Communication, 1969, $156 \mathrm{p}$.

2. Ginsburg OT. The propagation of electromagnetic waves in the plasma. Moscow, Science, 1967, 684 p.

3. Dolukhanov MP. The propagation of radio waves. M., State. Communications and Radio Publishing House, 1960, 336 p.

4. Yakovlev OI, Yakubov VP, Uryadov VP, Pavelev AG. The propagation of radio waves. M., LENAND, 2009, 496 c.

5. Sklyar B. Digital communication. Theoretical foundations and practical application. M., Publishing House "Williams", 2003, 1104 p.

6. Armand ON. Distribution of broadband signals in dispersive environments. Radio engineering and electronics, 2003, 48 (9): 1045-1057.

7. Ivanov DV, Ivanov VA, Mikheeva NN, Ryabov NV, Ryabov MI. Distribution of shortwave spread spectrum signals in a medium with nonlinear dispersion. Radio engineering and electronics, 2015, 60 (11): 1167-1177.

8. Hofinann-Wellenhof B, Lichtenegger H, Collins J. Global Positioning System. Theory and Practice. Springer-Verlag, New York, 1994, 392 p.

9. Kutuza BG, Moshkov AV, Pozhidaev VN. Combined method that eliminates the influence of the ionosphere when processing signals of P-range airborne radars with synthetic aperture. Radio engineering and electronics, 2015, 60 (9): 889-895.

10. Specification for 2-generation COSPASSARSAT $406 \mathrm{MHz}$ distress beacons. C / S T.018. Issue 1. 2016.

11. Bilitza D, McKinnell LA, Reinisch B, Fuller-Rowell T. The International Reference Ionosphere (IRI) today and in the future. Journal of Geodesy, 2011, 85: 909-920.

12. Nazarov LE, Batanov VV. Probabilistic characteristics of the detection of radio pulses during the propagation of satellite communication systems over the ionospheric transmission lines. Technology and Electronics, 2017, 62 (9): 866-874.

13. Dvorak SL, Dudley DG. Propagation of Ultrawideband Electromagnetic Pulses Through Dispersive Media. 
IEEE Transactions on Electromagnetic Compatibility, 1995, 37 (2): 192-200.

14. Nazarov LE, Batanov VV, Zudilin AS. Distortion of radio pulses during the propagation of satellite communication systems through the ionospheric lines. Journal of Radio Electronics, 2016, №2. Access mode: http://jre.cplire.ru/jre/ feb16/1/text.pdf.

15. Nazarov LE, Batanov VV. Analysis of distortions of radio pulses during the propagation of satellite communication systems over ionospheric transmission lines. Electromagnetic waves and electronic systems, 2016, 21 (5): 37-45. 